\title{
MODELOS DO PLANO PROJETIVO NO ESPAÇO TRIDIMENSIONAL
}

\author{
TON MARAR
}

\begin{abstract}
Resumo. Os bordos de um 2-disco e de uma faixa de Möbius são curvas fechadas simples. Quando identificamos ponto-a-ponto essas duas curvas, a superfície sem bordo resultante é um modelo do plano projetivo real $\mathbb{P}^{2}$. Vamos apresentar três modos de fazer esta identificação e obter três modelos de $\mathbb{P}^{2}$ em $\mathbb{R}^{3}$, topologicamente distintos. Tais modelos aparecem na literatura com a denominação: esfera com cross-cap, superfície romana de Steiner e superfície de Boy, porém sem os detalhes geométricos que apresentamos aqui.
\end{abstract}

\section{INTRODUÇÃO}

Hilbert \& Cohen-Vossen no clássico Geometry and the Imagination ([7] p.116), introduzem o plano projetivo como o plano euclidiano aumentado pela adição dos pontos no infinito. Cada ponto no infinito é a direção de uma reta. Assim, retas paralelas têm um ponto em comum, no infinito. Uma reta com seu ponto no infinito é chamada reta projetiva. Movendo-se ao longo de uma reta projetiva de um lado para o outro ou vice-versa somos levados ao mesmo ponto no infinito. Portanto, um círculo é um modelo geométrico da reta projetiva.
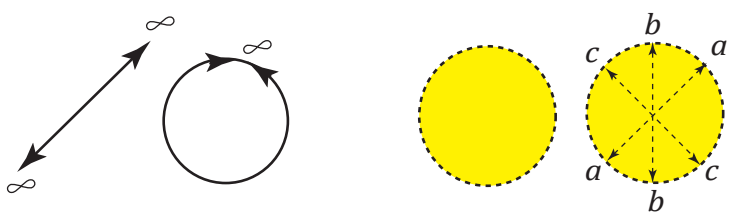

\section{FIGURA 1}

Analogamente, um modelo geométrico do plano projetivo é um 2-disco aberto, representando o plano euclidiano, aumentado com todos os seus pontos no infinito, isto é, as direções de todas as retas no plano. As direções das retas que passam pelo centro do disco correspondem a todos os pontos no infinito do plano; de fato,

Data de aceitação: Janeiro de 2021. 
qualquer reta no plano tem uma paralela passando pelo centro do disco. Cada uma dessas retas passando pelo centro do disco tem um par de pontos antipodais no bordo do disco, e eles devem ser identificados. Portanto, o disco com esses pares de pontos antipodais identificados é um modelo geométrico do plano projetivo (Fig.1).

Alternativamente, o plano projetivo é obtido geometricamente de uma faixa de Möbius e um 2-disco identificados ao longo do bordo.

Para ver isso, considere o disco e os seus pontos de bordo antipodais que serão identificados. Dividimos o disco em três partes, a saber, uma parte central e os setores à esquerda e direita (Fig.2 (a)). É fácil ver que depois da identificação dos antipodais, a parte central dará origem a uma faixa de Möbius. O restante do disco, os dois setores, depois de identificados ao longo do arco tracejado, desde $a$ até $c$, é homeomorfo a um novo disco cujo bordo é exatamente o bordo da faixa de Möbius.

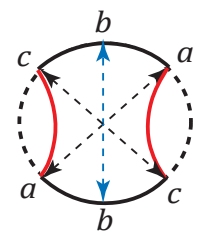

(a)

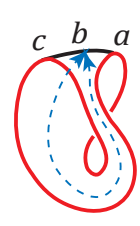

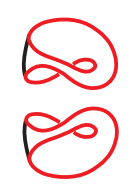

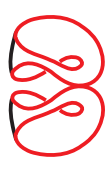

(b)

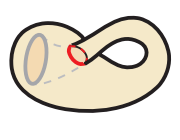

(b)

FIGURA 2

De modo mais geral, o espaço projetivo $n$-dimensional $P^{n}(E)$ associado ao espaço vetorial $E$ de dimensão $n+1$ sobre um corpo $K$ é o conjunto dos subespaços unidimensionais de $E$. Existe uma aplicação natural $p: E-\{0\} \rightarrow P^{n}(E)$ que associa cada vetor não nulo $\mathbf{u}$ ao subespaço gerado por $\mathbf{u}$. Uma topologia pode ser dada a $P^{n}(E)$ como a topologia quociente da aplicação natural $p$. Em particular, o plano projetivo real $\mathbb{P}^{2}$ é o conjunto de todas as retas que passam pela origem do $\mathbb{R}^{3}$.

Outra superfície obtida de faixas de Möbius é a garrafa de Klein. Um modelo da garrafa de Klein é construído identificando, ponto-a-ponto, os bordos de duas faixas (Fig.2(b)).

Garrafa de Klein e plano projetivo são exemplos de superfícies finitas em tamanho e sem bordo. Tais superfícies são chamadas superfícies fechadas.

O resultado da classificação topológica das superfícies fechadas foi publicado na Princeton Ph.D. Thesis de Henry Brahana em 1920 ([3]). Existe uma certa disputa de quem foi o primeiro a obter este resultado ([10] p. 53), fato não incomum em matemática.

A lista das superfícies fechadas se divide em duas, a saber, as superícies orientáveis e as não-orientáveis.
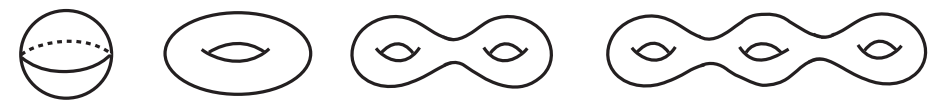

FIGURA 3

Modelos no $\mathbb{R}^{3}$ de superfícies orientáveis, como a esfera, o toro, bitoro, tritoro etc, dividem o espaço tridimensional em interior e exterior (Fig.3). 
Modelos de superfícies não-orientáveis no $\mathbb{R}^{3}$ são mais complicados. Enquanto superfícies orientáveis podem ser modeladas no $\mathbb{R}^{3}$ sem auto-interseções, qualquer modelo de superfície não-orientável fechada no $\mathbb{R}^{3}$ apresenta auto-interseções. Este é um corolário de um teorema de L. Brouwer, que pode ser lido assim:

Nenhum subconjunto fechado do espaço euclidiano n-dimensional é homeomorfo a uma $(n-1)$-variedade não-orientável ([5]).

No caso do plano projetivo, o processo para a obtenção do modelo geométrico identificando os bordos da faixa de Möbius e do 2-disco não é trivial. De fato, enquanto o bordo da faixa dá duas voltas até se fechar, o bordo do disco fecha depois de apenas uma volta. Assim, para que a identificação dos bordos ocorra no $\mathbb{R}^{3}$, é necessário permitir auto-interseções adequadas na faixa, como veremos na próxima seção.

A curva central da faixa de Möbius, conhecida como alma da faixa, é chamada caminho desorientador. Se percorrermos a faixa ao longo da sua alma, retornaremos ao ponto inicial de cabeça para baixo (ignorando a gravidade!) (Fig. 4(a)).

Diferente de um cilindro que possui dois lados, isto é, interior e exterior podem ser definidos, a faixa de Möbius possui apenas um lado.

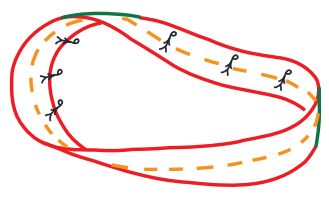

(a)

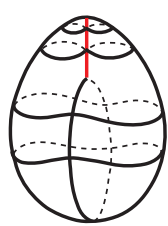

(b)

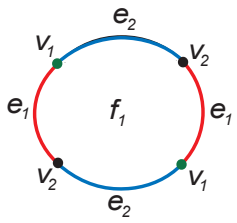

(c)

FIGURA 4

A presença de um caminho desorientador caracteriza a não-orientabilidade, isto é, uma superfície é não-orientável se, e somente se, contém uma faixa de Möbius.

Enquanto as superfícies orientáveis fechadas dividem o $\mathbb{R}^{3}$ em interior e exterior, as não-orientáveis fechadas, como o plano projetivo e a garrafa de Klein, não permitem a definição de lado de dentro e lado de fora. De fato, qualquer superfície mergulha no $\mathbb{R}^{4}$, isto é, podem ser representadas sem auto-interseção. No caso das superfícies não-orientáveis fechadas, a presença de uma faixa de Möbius, que possui apenas um lado, impossibilita a definição de interior e exterior.

É importante distinguir a superfície abstrata de seu modelo no $\mathbb{R}^{3}$. Auto-interseções presentes nos modelos de superfícies não-orientáveis no $\mathbb{R}^{3}$ permitem definir um interior e um exterior, lado de dentro e lado de fora, para os modelos de superfícies não-orientáveis. Além disso, as possíveis auto-interseções de um modelo podem afetar o tipo topológico do subconjunto do $\mathbb{R}^{3}$ que o representa. Esta diferença é percebida no cálculo da característica de Euler, isto é, a soma alternada do número de 0-células (vértices), 1-células (arestas) e 2-células (faces) de uma decomposição celular do modelo.

Hilbert \& Cohn-Vossen ([7], p.315) descrevem o modelo de $\mathbb{P}^{2}$ no $\mathbb{R}^{3}$ representado aqui na Fig.4(b), e afirmam que este modelo é topologicamente equivalente ao plano projetivo. Contudo, é fácil ver que a característica de Euler do modelo é igual a 2, 
enquanto tomando uma decomposição celular do plano projetivo (Fig. 4(c)), com dois vértices $v_{1}$ e $v_{2}$, duas arestas $e_{1}$ e $e_{2}$ e uma face $f_{1}$, obtém-se o valor 1 para a característica de Euler de $\mathbb{P}^{2}$. Portanto, o modelo do plano projetivo no $\mathbb{R}^{3}$ (Fig. $4(b))$ e a superfície abstrata $\mathbb{P}^{2}$ não são topologicamente equivalentes.

\section{TRÊS MODELOS DE $\mathbb{P}^{2}$ NO $\mathbb{R}^{3}$}

A esfera com cross-cap, a superfície romana de Steiner e a superfície de Boy, representadas respectivamente pelas Fig.5(a), Fig.5(b) e Fig.5(c), são os três modelos do plano projetivo no espaço tridimensional mais conhecidos na literatura.

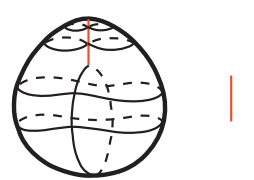

(a)

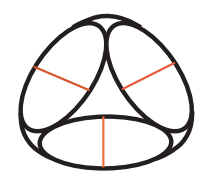

(b)
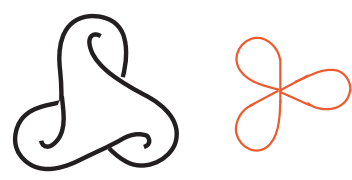

(c)

FIGURA 5

São obtidos da identificação do bordo de uma faixa de Möbius com o bordo de um 2-disco, porém com diferentes auto-interseções que permitem tal identificação no $\mathbb{R}^{3}$. A esfera com cross-cap tem um segmento de auto-interseção com dois pontos singulares nas extremidades. A superfície romana de Steiner tem três segmentos não coplanares de auto-interseção e cada um deles possui dois pontos singulares nas extremidades; além disso, os três segmentos se interceptam transversalmente num ponto triplo. A auto-interseção da superfície de Boy é uma rosácea de três pétalas não coplanares, que se interceptam transversalmente num ponto triplo.

Se considerarmos os modelos como imagem de aplicações $f: \mathbb{P}^{2} \rightarrow \mathbb{R}^{3}$ diferenciáveis, então a esfera com cross-cap e a superfície romana terão pontos onde a matriz jacobiana de $f$ tem posto igual a 1, pontos singulares. A superfície de Boy é a imagem de uma imersão, portanto posto constante e igual a 2.

H. Whitney ([12]) demonstrou que uma parametrização local dos pontos singulares que ocorrem nesses modelos é dada por $(x, y) \mapsto\left(x, y^{2}, x y\right)$. A imagem desta aplicação é conhecida como guarda-chuva de Whitney. A razão deste nome é que a superfície imagem, vista como solução da equação algébrica $Z^{2}-Y X^{2}=0$, inclui todo o eixo $Y$ (Fig.6), como o cabo de um guarda-chuva!

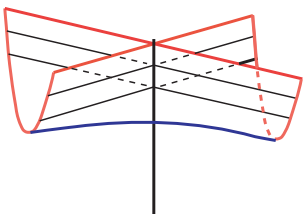

FIGURA 6

2.1. A esfera com cross-cap. Os pontos de $\mathbb{P}^{2}$ são as retas pela origem de $\mathbb{R}^{3}$, ou equivalentemente, são os pares de pontos antipodais de uma esfera com centro na origem de $\mathbb{R}^{3}$, isto é, cada reta pela origem do $\mathbb{R}^{3}$ determina um par de pontos naquela esfera e esses devem ser identificados. 
Faremos a identificação dos antipodais de uma esfera por partes. Inicialmente dividimos a esfera em equador e dois hemisférios, um superior e outro inferior. Identificamos todos os pontos do hemisfério superior com os pontos do inferior após uma rotação de $180^{\circ}$. Em seguida identificamos os pontos antipodais do equador (Fig.7).
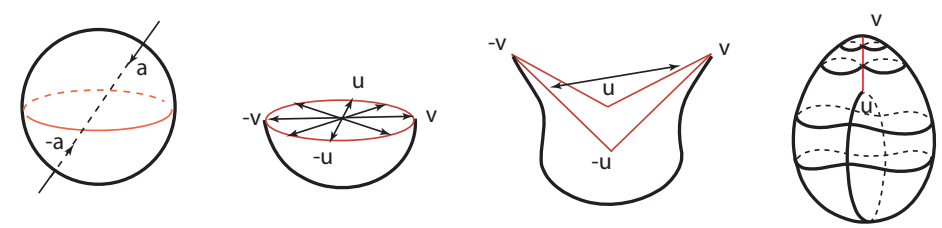

FIGURA 7

Esta construção encontra-se em Hilbert \& Cohn-Vossen ([7], p. 314).

Uma construção mais explícita da esfera com cross-cap pode ser feita usando uma adequada projeção no $\mathbb{R}^{3}$ de um mergulho da faixa de Möbius no $\mathbb{R}^{4}$.

Considere uma faixa de Möbius mergulhada no $\mathbb{R}^{4}$ e sua alma obtida da interseção com um plano transversal à faixa. Tomemos a projeção $\mathbb{R}^{4} \rightarrow \mathbb{R}^{3}$ que envia todo o plano contendo a alma sobre uma reta no $\mathbb{R}^{3}$. Esta projeção identifica dois-a-dois os pontos da alma, enviando-a sobre um intervalo de reta com dois pontos singulares como extremos. Esses pontos singulares são precisamente a imagem dos pontos da alma onde a direção de projeção é tangente à faixa.
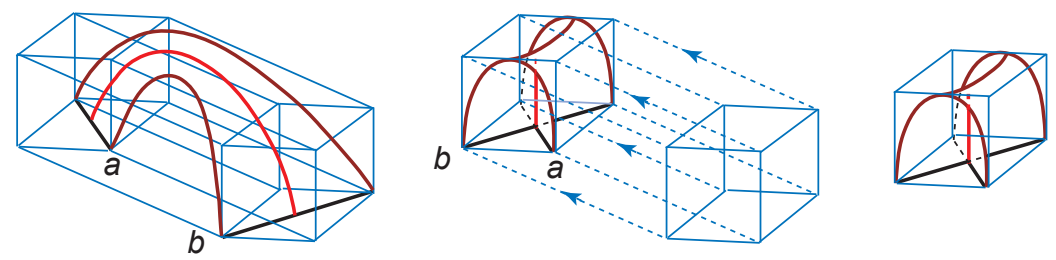

FIGURA 8

A figura 8 descreve a projeção de um hipercubo, contendo meia faixa de Möbius, sobre um cubo que contém um guarda-chuva de Whitney, imagem da projeção da meia faixa.

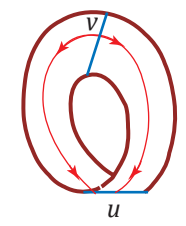

(a)

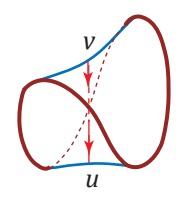

(b)

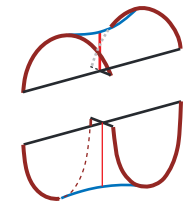

(c)

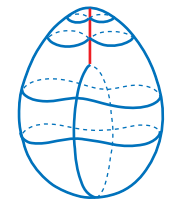

(d)

FIGURA 9

A imagem de toda a faixa de Möbius (Fig.9(a)) por esta projeção é chamada conóide de Plücker (Fig.9(b)). Enquanto o bordo da faixa dá duas voltas até se fechar, o bordo do conóide é um bordo circular, fecha depois de apenas uma volta. 
Assim, a identificação no $\mathbb{R}^{3}$ dos pontos do bordo do conóide com os pontos do bordo do 2-disco é direta. O resultado é o modelo esfera com cross-cap do plano projetivo (Fig.9(d)).

É fácil ver que o conóide é obtido da identificação de dois guarda-chuvas de Whitney (Fig.9(c)), ambos projeções, do $\mathbb{R}^{4}$ no $\mathbb{R}^{3}$, de meia faixa de Möbius, sendo um rotacionado de $\pi / 2$ em relação ao outro. Isso simplifica o entendimento dos dois pontos singulares do modelo esfera com cross-cap (Fig.9(d)).

Também é fácil ver que o conóide (Fig.9(b)) é contrátil a um ponto. Assim, o modelo esfera com cross-cap (Fig.9(d)) tem característica de Euler igual a 2.

2.2. Superfície romana de Steiner. A superfície romana é um modelo de $\mathbb{P}^{2}$ no $\mathbb{R}^{3}$ cuja auto-interseção se dá ao longo de três segmentos não coplanares que se cruzam em um ponto triplo. Nas extremidades dos três segmentos, o modelo possui seis pontos singulares do tipo guarda-chuva de Whitney. Supostamente Jakob Steiner (1796-1863) encontrou este modelo quando esteve em férias em Roma em 1844.

Hilbert \& Cohn-Vossen ([7] p.302) fazem uma descrição poliedral deste modelo que denominam heptaedro. Também se sabe que esta superfície é uma superfície algébrica dada pela equação quártica $x^{2} y^{2}+x^{2} z^{2}+y^{2} z^{2}-x y z=0$.

Assim como fizemos com o cross-cap, usaremos uma projeção adequada da faixa de Möbius do $\mathbb{R}^{4}$ no $\mathbb{R}^{3}$ para facilitar a visualização da superfície romana.
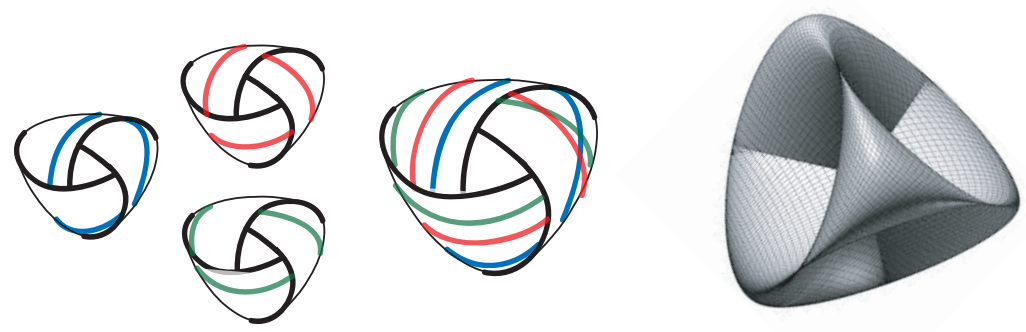

FIGURA 10

Desta vez porém consideramos três curvas em três planos distintos, representando três caminhos desorientadores da faixa, e de modo que esses caminhos se cruzam aos pares (Fig.10). Com esta escolha, após a projeção do $\mathbb{R}^{4}$ no $\mathbb{R}^{3}$, cada um dos três planos é projetado em uma reta e essas três retas são não coplanares.

Esta projeção do $\mathbb{R}^{4}$ no $\mathbb{R}^{3}$ é uma generalização da projeção do $\mathbb{R}^{3}$ no $\mathbb{R}^{2}$ que leva dois planos em duas retas (Fig.11). O $\mathbb{R}^{4}$ é amplo o suficiente para conter os três planos que são projetados em três retas não coplanares no $\mathbb{R}^{3}$.

Além disso, cada um dos três caminhos desorientadores é projetado em um segmento que tem dois pontos singulares nas extremidades e os três possuem um ponto em comum.

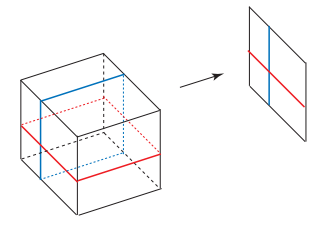

FIGURA 11 De fato, uma vizinhança de cada um dos três caminhos 
desorientadores, isto é, uma faixa de Möbius, é projetada no $\mathbb{R}^{3}$ num conóide de Plücker (Fig.9(b)).

Na Fig.12(a) os três caminhos são desenhados num modelo plano da faixa de Möbius. Três pequenos discos são removidos para facilitar o entendimento da projeção. A projeção terá quatro componentes de bordo, três correspondentes aos discos removidos e um que é o bordo da faixa.

A Fig.12(b), composição de três conóides foi desenhada por Scott Carter ([6] p.202), baseado no que ele denomina Marar's models of singular surfaces.

Observe que podemos retrair as quatro componentes de bordo até os segmentos de auto-interseção e esses a um ponto. Portanto, a superfície Fig.12(b) é contrátil e o modelo da superfície romana é obtido colando-se os quatro discos ao longo das suas componentes de bordo. Portanto sua característica de Euler é igual a 5.

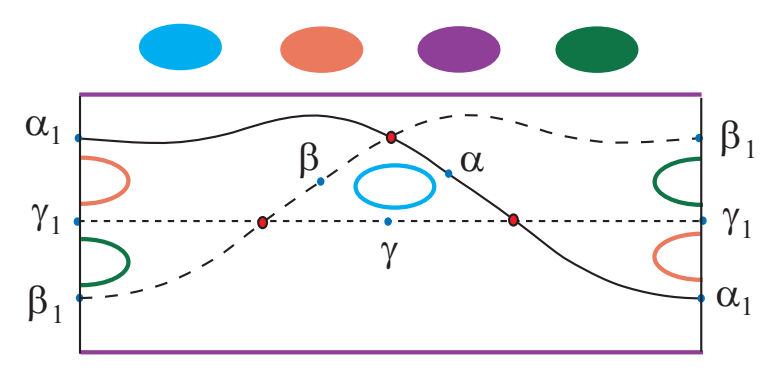

(a)

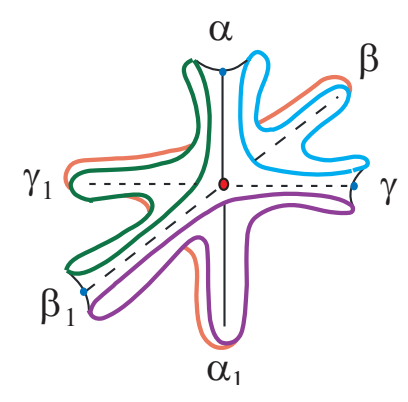

(b)

FigurA 12

2.3. A superfície de Boy. Esta superfície é um modelo de $\mathbb{P}^{2}$ no $\mathbb{R}^{3}$ com um único ponto triplo. Foi descoberto em 1901 por Werner Boy (1879-1914). O modelo não possui pontos singulares, é uma imersão de $\mathbb{P}^{2}$ no $\mathbb{R}^{3}$. François Apéry ([1]) encontrou uma parametrização dessa imersão, assim como uma equação algébrica de grau seis definindo a superfície de Boy.

Um corolário de um teorema de Thomas Banchoff ([2]) assegura que toda imersão de $\mathbb{P}^{2}$ no $\mathbb{R}^{3}$ possui um número impar de pontos triplos. De fato, Banchoff mostra que a característica de Euler $\chi(S)$, de uma superfície fechada $S$, tem a mesma paridade que o número de pontos triplos de qualquer imersão de $S$ no $\mathbb{R}^{3}$. No caso de $\mathbb{P}^{2}$, a caracteristica de Euler $\chi\left(\mathbb{P}^{2}\right)=1$, e assim, um número impar de pontos triplos ocorre em qualquer imersão de $\mathbb{P}^{2}$ no $\mathbb{R}^{3}$.

Werner Boy foi aluno de doutorado de David Hilbert. Alguns dizem que Hilbert pediu para Boy demonstrar que qualquer modelo de $\mathbb{P}^{2}$ no $\mathbb{R}^{3}$ possui no mínimo dois pontos singulares. Contudo, a superfície de Boy é um contraexemplo a esta suposta conjectura de Hilbert. No prefácio do livro de Apéry ([1]), Egbert Brieskorn escreve: ... the famous mathematician David Hilbert was mistaken.

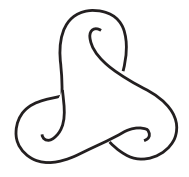

FigurA 13 
A projeção sobre $\mathbb{R}^{3}$ do mergulho da faixa de Möbius no $\mathbb{R}^{4}$ que mostramos na Fig.8, exibe inevitáveis pontos de tangência da direção de projeção com a faixa. De fato, uma conjectura de Whitney de 1940, demonstrada por William Massey ([11]), tem como corolário que toda projeção sobre $\mathbb{R}^{3}$, de uma mergulho de $\mathbb{P}^{2}$ no $\mathbb{R}^{4}$, possui no mínimo dois pontos singulares.

Portanto, Boy mergulhou a faixa de Möbius no $\mathbb{R}^{5}$ e projetou diretamente no $\mathbb{R}^{3}$, evitando qualquer ponto de tangência com a direção de projeção.

A tese de doutorado de Boy foi publicada em 1903 ([4]) e contém belíssimos desenhos e fotos de modelos de sua imersão de $\mathbb{P}^{2}$ no $\mathbb{R}^{3}$. Este é o único registro acadêmico deixado por Boy que, como soldado na Primeira Guerra Mundial, foi abatido logo no seu início, em 6 de setembro de 1914.
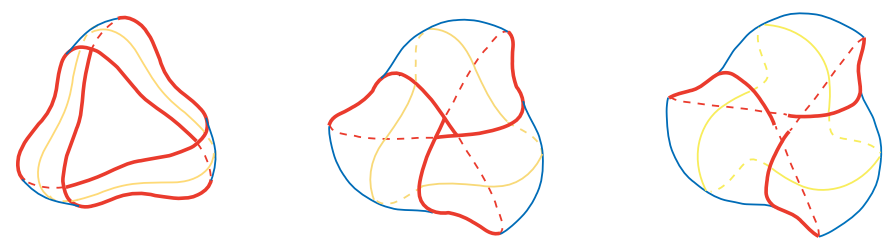

FIGURA 14

Para ver a superfície de Boy como uma faixa de Möbius com um disco colado ao longo de seu bordo, deformamos a faixa (Fig.14) criando um ponto triplo (Fig.15). Este é um processo análogo ao que fizemos na deformação da faixa de Möbius quando criamos o conóide de Plücker (Fig.9(b)).
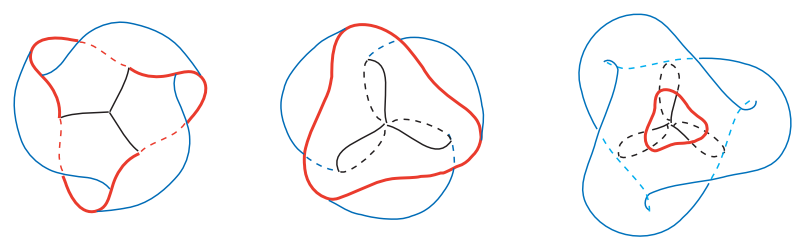

FIGURA 15

A característica de Euler da superfície de Boy é igual a 2, mas isso não é fácil de ver. Neste caso, uma fórmula do tipo Riemann-Hurwitz, como explicitaremos abaixo, pode ajudar.

O conceito de aplicação semiregular $f: N^{n} \rightarrow \mathbb{R}^{2 n-1}$, onde $N^{n}$ é uma variedade $n$-dimensional, $n \geq 2$, foi introduzido por Whitney no artigo de 1944 publicado no Annals of Mathematics ([12]). No caso $n=2$, uma aplicação semiregular de uma superfície com imagem no $\mathbb{R}^{3}$ é uma imersão, a menos de um número finito de pontos singulares do tipo guarda-chuva de Whitney.

Se no $\mathbb{R}^{3}$ considerarmos o modelo $M$ de uma superfície $S$ como a imagem de uma aplicação semiregular então as características de Euler $\chi(M)$ de $M$ e $\chi(S)$ de $S$ são relacionadas por uma fórmula, obtida por S. Izumyia e o autor ([8]).

Teorema. Seja $f: S \rightarrow \mathbb{R}^{3}$ uma aplicação semiregular de uma superfície fechada $S$ e cuja imagem $f(S)=M$ é um modelo de $S$ no $\mathbb{R}^{3}$. Sejam $T$ e $C$ respectivamente 
o número de pontos triplos e guarda-chuvas de Whitney do modelo M. Então,

$$
\chi(M)=\chi(S)+T+C / 2 .
$$

Como a superfície $S$ é fechada, o número $C$ é par. De fato, os pontos singulares (guarda-chuvas de Whitney) são os pontos iniciais (ou finais) da curva de autointerseção do modelo $M$. Assim, como a superfćie é fechada, a presença de um guarda-chuva (ponto inicial) requer a presença de outro (ponto final).

Esta fórmula facilita o cálculo da diferença das características de Euler da superfície abstrata e de seu modelo no $\mathbb{R}^{3}$. Por exemplo, como $\chi\left(\mathbb{P}^{2}\right)=1$, a fórmula nos dá imediatamente a caracteística de Euler da esfera com cross-cap, superfície romana e superfície de Boy, a saber, 2, 5 e 2. De fato, a esfera com cross-cap tem $(C, T)=(2,0)$, a superfície romana tem $(C, T)=(6,1)$ e a superfície de Boy tem $(C, T)=(0,1)$.

Agradecimentos. Agradeço ao referee anônimo e aos meus colegas François Apéry, J. Scott Carter e Thomas Banchoff por sugestões que melhoraram o texto.

\section{REFERÊNCIAS}

[1] Apéry, F.; Models of the real projective plane, Vieweg 1987.

[2] Banchoff, T. F.; Triple points and surgery of immersed surfaces; Proc. Amer. Math. Soc. 46 (1974) 407-413.

[3] Brahana, H. R. ; Systems of circuits on two-dimensional manifolds; Annals of Mathematics 23 (1921) 144-168.

[4] Boy, W.; Über die Curvatura integra und die Topologie geschlossener Flächen, Mathematische Annalen 57 (1903) 151-184.

[5] Brouwer, L.E.J.; Sur le théorème de M. Jordan dans l'espace à n dimensions, Comptes Rendus Paris 153 (1911) 542-544.

[6] Carter, J. Scott; How surfaces intersect in space, Second edition, World Scientific 1995.

[7] Hilbert, D. \& Cohn-Vossen, S. ; Geometry and the imagination, Chesea 1952 (a translation of Anschauliche geometrie, Springer 1932).

[8] Izumyia, S. \& Marar, W. L.; On topologically stable singular surfaces in a 3-manifold, Journal of Geometry 52 (1995) 108-119.

[9] Marar, Ton; Topologia geométrica para inquietos, Edusp 2019.

[10] Massey, W. S.; Algebraic Topology: An Introduction, Springer 1967.

[11] Massey, W. S.; Proof of a conjecture of Whitney, Pacific J. Math. 31 (1969) 143-156.

[12] Whitney, H.; The singularities of a smooth $n$-manifold in $(2 n-1)$-space, Annals of Mathematics 45 (1944) 247-293.

Departamento de Matemática Aplicada e Estatística Instituto de CiênCias Matemáticas E de ComputaÇÃo Universidade de SÃo PAUlo

EMAIL:TON@ICMC.USP.BR 